\title{
Web crawling and mRNA sequencing analyze mechanisms of photobiomodulation
}

\author{
Hao Wu \\ Harbin Medical University \\ Caiyun Meng \\ Harbin Medical University \\ Qing Xia \\ Harbin Medical University \\ Hailiang Liu \\ Harbin Medical University \\ Fengmin Zhang \\ Harbin Medical University \\ Wuqi Song ( $\square$ songwuqi@hrbmu.edu.cn ) \\ Harbin Medical University https://orcid.org/0000-0001-5900-5154
}

\section{Research}

Keywords: photobiomodulation, web crawling, mRNA sequence, mechanisms, key genes, RT-qPCR.

Posted Date: June 24th, 2021

DOI: https://doi.org/10.21203/rs.3.rs-646073/v1

License: (c) (i) This work is licensed under a Creative Commons Attribution 4.0 International License.

Read Full License

Version of Record: A version of this preprint was published at Photobiomodulation, Photomedicine, and Laser Surgery on April 1st, 2022. See the published version at https://doi.org/10.1089/photob.2021.0142. 


\section{Abstract}

Background Photobiomodulation (PBM) is praised as a promising physical therapy, which has many advantages, such as noninvasive, painless. However, the mechanisms are not fully elucidated.

Methods Using web crawling, mRNA sequence and bioinformatics analysis to select genes, functional annotation, and mechanisms. The expressions of inflammatory cytokines were measured using RTqPCR.

Results A total of 146 human genes and 57 pathways were identified about PBM. The 630nm LEDstimulated-MH7A cells were sequenced for further analyzing the mechanism of PBM. 2950 differentially expressed genes were identified, and the gene ontology term enrichment analysis and Kyoto encyclopedia of genes and genomes pathway analysis were performed to better understand functions and pathways. The 12 pathways were matched with the KEGG results of PBM and MH7A cells. A protein-protein interaction network was performed among genes in 12 pathways, and 10 outstanding proteins were identified. Importantly, the 9 genes were predicted with potential research value.And we also demonstrated that expression of inflammatory factors (IL-6, IL-1 $\beta$, IL-8, and MMP-3) was reduced; meanwhile, the expression of anti-inflammatory factor IL-10 was promoted after $630 \mathrm{~nm}$ LED. In conclusion, using web crawling, bioinformatics analysis, and mRNA sequence, we obtained 9 key genes and 10 important pathways about PBM. Importantly, we demonstrated the anti-inflammatory effect of 630nm LED red light by RT-qPCR.

\section{Introduction}

PBM is a new physical therapy, and it utilizes endogenous chromophores with low dose illumination delivered at the target site, which is the opposite of photodynamic therapy by stimulating exogenous chromophore ${ }^{[1]}$. PBM is also called low-level laser therapy(LLLT), which is a non-invasive form of phototherapy that utilizes wavelengths of light between 650 and $1000 \mathrm{~nm}$ to deliver low irradiance and doses to the target tissue ${ }^{[2]}$. So far, low-level laser therapy has been recognized as a light therapy that may be used for anti-inflammation, promoting wound healing and bone regeneration ${ }^{[3-5]}$.

Although many articles have put forward many opinions, the mechanisms of PBM are not fully elucidated. Studies have demonstrated that NF-kB was significantly activated on $0.003 \mathrm{~J} / \mathrm{cm}^{2}$ by irradiating rat embryonic fibroblasts with $810 \mathrm{~nm}$ laser ${ }^{[6]}$. Some studies have found that LLLT can inhibit apoptosis thl rough the PI3K-AKT signaling pathway. LPLI inhibits staurosporine (STS)-induced cell apoptosis by inactivating the GSK-3b/Bax pathway ${ }^{[7]}$. However, it has also been demonstrated that LLLT enhances the invasivity of tumor cells by regulating the Akt/ mTOR signaling pathway ${ }^{[8]}$.

Photodynamic therapy(PDT) is a highly effective and safe treatment method of phototherapy ${ }^{[9]}$. Photodynamic therapy is firstly reported in the 1970 s and is honored as the most promising treatment model of the 20 th century in the $1980 \mathrm{~s}^{[10]}$. It used chemical reactions between light and photosensitizers 
in specific wavelengths to produce reactive oxygen species that kill target cells for therapeutic effect ${ }^{[11]}$. Both light and photosensitizers were harmless and can be activated when exposed to a specific wavelength of light in a specific area. Therefore, this treatment has many advantages, including strong controllability, low toxicity, and non-invasive, etc ${ }^{[12]}$. To demonstrate whether the mechanism of PBM and PDT is different, this study compares the similarities in the genes and pathways affected by PBM and PDT.

Light sources are laser and light-emitting diode(LED) in phototherapy. LED, as the fourth generation light source, has the advantages of energy-saving, environmental protection, and high electro-optical conversion efficiency. Therefore, LED is becoming more application in LLLT. However, the two light sources have different properties ${ }^{[13]}$. Due to the laser is coherent light, but LED is incoherent light, thus one of our aims is to compare the effects of laser and LED.

In conclusion, we crawled 3352 literature, identified 146 human genes from the abstracts of the literature, and performed bioinformatic analyses to get 9 key genes and important 12 pathways about PBM.

\section{Methods}

\section{Data collection}

\subsection{Crawling literature on photobiomodulation and photodynamic therapy}

The research design was created in the form of a flowchart(Figure 1). In the NCBI PubMed database, with 'photobiomodulation' or 'low level laser therapy' as keywords in [Title and Abstract] to search PBM-related literature. The same way that keywords were 'photodynamic therapy' to search PDT-related literature. And crawled through $\mathrm{R}$ language to get the abstracts of the relevant literature.

\subsection{Crawling LED, laser-related literature}

In the NCBI PubMed database, due to the research on LED started late, there were few articles related to it. To expand the retrieval range, we searched relevant literature directly with the 'light-emitting diode' as a keyword. Similarly, in [title and abstract], 'laser' was used as the keyword to search laser-related literature. And crawled literature through $\mathrm{R}$ language to get the abstracts of the relevant literature.

\section{Data analysis}

\subsection{Identification of genes in the relevant literature}

Adopting the gene_atomization function in the pubmed. mineR package of the $\mathrm{R}$ language, the genes in the abstracts in the relevant literature can be directly extracted. The gene_atomization can be used to identify genes in relevant literature abstracts with PBM, PDT, LED, and laser.

\subsection{Confirming the related genes of LED and laser phototherapy}


The genes of the PBM group, PDT group, LED group, and laser group was identified, and their respective intersections were obtained through the Venn diagram. The intersection between LED genes and (PBM+PDT) group genes was LED -related phototherapy genes. Similarly, laser-related phototherapy genes were confirmed.

\subsection{Enrichment of functions and pathways}

To study the genes that have been identified from the relevant literature, we carried out a comprehensive set of functional annotations for them and used the "Clusterprofiler" package for gene ontology (GO) term enrichment analysis and Kyoto genes and Encyclopedia of Genomics (KEGG) pathway analysis ${ }^{[14]}$. All KEGG pathway and GO enrichment analysis was based on a threshold of $p$-value $<0.05$.

\subsection{PPI network}

Search Tool for the Retrieval of Interacting Genes/Proteins (STRING) database (http://www.strin-db.org/) is a systems biology tool to assess protein-protein interaction (PPI) information ${ }^{[15]}$. To evaluate the interrelationships behind the genes, we used the STRING database for analysis. Meanwhile, the MCODE [16] plug-in of Cytoscape software was used to visualize and select hub modules of the PPI network. The degree $=5$, node score $=0.2$, $k$-core $=2$, and maximum depth $=100$ were used as cut-off criteria .

\section{Cell culture}

Human fibroblast-like synoviocyte line, MH7A was purchased from GuanDao Biological Research Center of Shanghai. Cells were cultured in DMEM supplemented with fetal bovine serum (10\%) and penicillin/streptomycin solution(1\%). The cultured cells were maintained at $37^{\circ} \mathrm{C}$ in a $5 \% \mathrm{CO} 2$ atmosphere.

\section{Light-emitting diode bio-stimulation}

Light-emitting diodes (CP-YL3213-630, Truwin Optoelectronic Medical, Beijing, China) were used to deliver 630-nm radiation. Cells were exposed through the bottom of plastic culture plates to the $630-\mathrm{nm}$ radiation. The time it takes to radiation once was $15 \mathrm{~min}$ to achieve radiation energies of $39 \mathrm{~J} / \mathrm{cm}^{2}$. TNF-a stimulation groups in which TNF-a $(10 \mathrm{ng} / \mathrm{mL}$, PeproTech) was added before the radiation. Cells of experimental groups were radiated at room temperature. The control groups (non-irradiated cells) were wrapped with aluminum foil and kept at room temperature while the experimental samples were radiated.

\section{Gene sequencing}

mRNA-seq was performed as reported in Genesky Biotechnologies Inc., Shanghai, China. We used the "limma" R package to screen the differentially expressed genes between LED+TNF-a samples and normal samples ${ }^{[17]}$. $P$-value $<0.05$ and $\mid \log 2$ fold change $(F C) \mid>1$, were chosen as the cut-off threshold.

\section{RT-qPCR}


Reverse transcriptase polymerase chain reaction (RT-PCR) was performed on total RNA harvested from synovioblasts with RNeasy mini kit (HaiGene, Harbin, China). Equal amounts of total RNA $(2 \mu \mathrm{g})$ was reverse transcribed by Thermo script RT-PCR system (Invitrogen, Carlsbad, CA). The resultant cDNA was PCR amplified for pro-inflammatory cytokines by using human gene-specific sense and antisense primers based on sequences published in GenBank: IL- 6 sense 5'-CCTGAACCTTCCAAAGATGGC-3', IL-6 antisense 5'-TTCACCAGGCAAGTCTCCTCA-3'; IL-8 sense 5'-ATGACTTCCAAGCTGGCCGTGGCT-3',IL-8 antisense 5'TCTCAGCCCTCTTCAAAAACTTCTC-3'; $\beta$-actin sense 5'-AAACTGGAACGGTGAAGGTG-3', $\beta$-actin antisense 5'-GTGGACTTGGGAGAGGACTG-3';IL-1 $\beta$ sense 5'-TGGCAATGAGGATGACTTGT-3', IL-1 $\beta$ antisense 5'TGGTGGTCGGAGATTCGTA-3'; IL-10 sense 5'-GACTTTAAGGGTTACCTGGGTTG-3'; IL-10 antisense 5'TCACATGCGCCTTGATGTCTG-3'. PCR was carried out on a LightCycler® 96 thermal cycler Instrument (Roche Applied Science, Penzberg, Germany). The program used for quantitative PCR amplification included a 5-min predenaturation step at $95^{\circ} \mathrm{C}$, a 3-step amplification at $95^{\circ} \mathrm{C}$ for $10 \mathrm{~s}, 60^{\circ} \mathrm{C}$ for $20 \mathrm{~s}$ (for 45 cycles), and a dissociation step $\left(10 \mathrm{~s}\right.$ at $98^{\circ} \mathrm{C}, 60 \mathrm{~s}$ at $65^{\circ} \mathrm{C}$, and $1 \mathrm{~s}$ at $\left.97^{\circ} \mathrm{C}\right)$.

\section{Results}

\section{Number of crawled literature}

A total of 3,352 literature related to photobiomodulation (PBM group), 20,442 literature related to photodynamic therapy (PDT group), 5,874 LED-related literature (LED group), and 326,426 articles on laser-related were crawled. (laser group).

\section{Gene identification}

A total of 146 human genes related to PBM, 268 human genes related to PDT were identified, and the frequency of top10 genes was shown in Figure $2 \mathbf{a}, \mathbf{2 b}$. The 142 human genes related to LED and 3188 human genes related to laser were identified.

\section{Confirm the genes in LED/laser-related phototherapy}

In this experiment, the intersected genes of the LED group genes and (PBM + PDT) group genes were LED-related phototherapy. In the same way, the genes in laser-related phototherapy were confirmed. By using the Venn diagram, as shown in Figure 3, it was clear that there were 161 human genes related to LED, of which 50 genes were intersected with PBM, 54 genes were intersected with PDT, and 29 genes were repeated intersecting. The 3188 human genes related to laser light therapy, of which 138 genes were intersected with PBM, 141 genes were intersected with PDT, and 59 genes were repeated intersecting. In summary, by crawling the literature and identifying genes, a total of 75 phototherapy-related genes with LED and 220 phototherapy-related genes with laser were found. Moreover, the genes were deserved to be attention in the public intersection of LED, laser, PBM, and PDT. We proposed that the genes in the public intersection may be the key genes that play a role in phototherapy. For this reason, enrichment analysis was performed on these genes. 


\section{Comparison of PBM and PDT}

In the R language, the clusterprofiler package was used to enrich functions and pathways. Among them, a total of 57 pathways in the PBM group were enriched, and the results of enrichment were shown in Figure $4 a$, $b$; a total of 109 pathways in the PDT group were enriched. The results of pathway and function enrichment were shown in Figure $\mathbf{5 a}$, b; Compare the two results, a total of 45 pathways were in the intersection, which intersection is $78.9 \%$ of the KEGG result of PBM.

\section{Comparison of LED and laser}

By crawling the literature and identifying genes, a total of 75 phototherapy-related genes with LED and 220 phototherapy-related genes with laser were found. No KEGG result in the LED-related phototherapy, which was caused by the number of identified genes was not enough. A total of 104 pathways were enriched in the laser group, as shown in Figure 6. However, it can be known that 74 genes were intersected with laser in 75 LED-related phototherapy genes from the Venn diagram. The intersected genes account for up to $98.7 \%$ of the 91 LED genes. Therefore, it can be concluded that the genes affected by the LED in phototherapy are the same as the laser light source.

\section{The 29 genes that were located in the common intersection of PBM, PDT, LED, and laser}

Known from the Venn diagram that a total of 29 genes were a common intersection in these four groups, and so we speculated that these genes may be the keys in phototherapy. Therefore, we also performed KEGG and GO analysis for these 29 genes. A total of 7 pathways were enriched. The results were shown in Figure 7a. At the same time, we also performed a GO enrichment analysis for these genes, and a total of 365 results were enriched, as shown in Figure 7b. It was obvious that these genes were related to tyrosine, ultraviolet, light, and oxygen stress, etc.

\section{Sequencing results of LED stimulate-MH7A}

To explore the mechanisms of PBM for RA disease, we sequenced LED-MH7A cells. First, MH7A cells were stimulated with TNF-a. The experimental groups were irradiated with $630 \mathrm{~nm}$ LED for $15 \mathrm{~min}$ at room temperature, while control groups were wrapped with aluminum foil at room temperature. A total of 2950 differentially expressed genes were identified. 1885 and 1065 differentially expressed genes were upregulated,down-regulated, respectively. The volcano is shown in Figure 8. KEGG enriched to 29 signal pathways, as shown in Figure 9. Among the 29 signaling pathways, 12 pathways were matched with those obtained by PBM analysis in this paper, which are Cytokine-cytokine receptor interaction, Legionellosis, Prion diseases, Viral protein interaction with cytokine and cytokine receptor, Complement and coagulation cascades, Bladder cancer, Fluid shear stress, and atherosclerosis, Prostate cancer, MAPK signaling pathway, TNF signaling pathway, IL-17 signaling pathway, Hepatitis B. All data, including sequencing reads and single-nucleus expression matrices have been deposited in NCBI's Gene Expression Omnibus (http://www.ncbi.nlm.nih.gov/geo/). The accession number for the mRNA-seq data reported in this study is GSE172223. 


\section{PPI network and cluster analysis}

For further analysis, we got 12 pathways that were matched in the KEGG results of MH7A and the KEGG results of PBM. The pathways were Cytokine-cytokine receptor interaction, Legionellosis, Prion diseases, Viral protein interaction with cytokine and cytokine receptor, Complement and coagulation cascades, Bladder cancer, Fluid shear stress and atherosclerosis, Prostate cancer, MAPK signaling pathway, TNF signaling pathway, IL-17 signaling pathway, Hepatitis B. The genes were constructed a PPI network in 12 pathways. The outcomes of the string profile obtained from STRING showed that contained 129 nodes and 1235 edges. The top 10 outstanding proteins were identified as hub genes, which might play critical roles in the PBM through PPI analysis of STRING software including IL6, TNF, VEGFA, IL 10, EGFR, EGF, CCL2, MMP9, IL 1B, FOS. Among these significant proteins, IL6 was the most important protein and connected with 84 nodes in the network. A total of 6 clusters were generated in MCODE, and the top one cluster was selected as hub modules by the scores evaluated in MCODE. MCODE 1, which had 32 nodes and 369 edges, had the highest score in these clusters, such as Figure 10. In this cluster, the 9 nodes, including IL6, VEGFA, CXCL2, CSF1P, ITGAM, TNF, FOS, MMP9, PGF were in both MH7A cells and the PBM group. Maybe, these 9 nodes are key genes for PBM. On the one hand, in this cluster, we can know that some protein(red circles) of PBM results obtained by the method proposed in this paper already coincide with the parts of bio-MH7A cell sequencing. It proves the feasibility of our method. On the other hand, in LED stimulate-MH7A cells, the yellow circles, including CSF3, CXCL3, SOCS3, HMOX1, HBEGF, IL1B, IL 1A, PLAU, and CCL20, represent the differentially expressed genes and have not been studied. Therefore, these genes have more research value and potential.

\section{Effects of 630-nm LED radiation on mRNA gene expressions of inflammatory cytokines}

For the accuracy of the PPI network and the effect of 640nm LED red light on rheumatoid disease, we performed RT-qPCR to analysis mRNA levels of inflammatory. The result was shown in Figure 11; there was a significant decrease in the gene expressions of inflammatory cytokines IL-6, IL-8, and IL-1Band MMP-3 expression; meanwhile, the expression of antiinflammatory factor IL-10 was promoted with the radiation doses increased. Collectively, these results demonstrate that the gene expression and production of inflammatory cytokines by MH7A cells can be inhibited by 630 -nm LED radiation.

\section{Discussion}

Although PBM has great potential applications, the mechanisms are not fully elucidated. By crawling the abstracts from the literature, the genes that have been studied were identified. And the enrichment analysis of KEGG and GO were adopted to know which pathways and functions were involved. Currently, most of the research on PBM is focuses on the PI3K-Akt signaling pathway and rheumatoid arthritis disease. At the same time, in the KEGG results of the laser group, the PI3K-Akt signaling pathway and rheumatoid arthritis signaling pathway appear again, which suggests that PBM regulates the PI3K-Akt and rheumatoid arthritis signaling pathway to play a role. Possibly, we put forward some hypotheses based on these results. Through PI3K-Akt and MAPK signaling pathways, rheumatoid arthritis could be 
improved by PBM. Coincidentally, one study has confirmed that $630 \mathrm{~nm}$ LED can inhibit the proliferation of synovial fibroblasts in rheumatoid arthritis through the PI3K-Akt signaling pathway ${ }^{[18]}$.

The PI3K-Akt signaling pathway is one of the most investigated pathways in PBM. The p53 and PI3-Akt signaling pathways mediate the up-regulation of GADD45 to induce human RPE cell apoptosis ${ }^{[19]}$; lowlevel laser therapy induces the proliferation, migration, and tube formation of human umbilical vascular endothelial cells by activating the PI3K/Akt signaling pathway ${ }^{[20]}$; LLLT reduces cytokines by inhibiting PI3K activation of protein kinase A16 ${ }^{[21]}$; LLLT (660 nm or $780 \mathrm{~nm}$ ) can modify oral dysplastic cells (DOK) and oral cancer cells (SCC9 and SCC25) growth by modulating the Akt/mTOR/CyclinD1 signaling pathway ${ }^{[8]}$.

A large number of studies have demonstrated that the MAPK signaling pathway plays a key role in PBM. 450nm PBM controls the bacterial infection of periodontitis through ROS/MAPK/mTOR pathway ${ }^{[22]}$. Studies have also shown that PBM can improve acute inflammation in mice through the cannabinoid receptor/ATP-sensitive K(+) pathway/P38-MAPK signaling pathway ${ }^{[23]}$. Melatonin-treated cells were laserirradiated, and the differentiation and mineralization of cells were found to involve p38 MAPK and PRKD1 signaling mechanisms ${ }^{[24]}$.

Crawling information through the Internet is a promising strategy. Georgia crawled obituaries online in 2015 to analyze the link between parity and cancer risk ${ }^{[25]}$. In our study, we used web crawling to obtain genes for comparing two different phototherapy methods: PBM and PDT. The result was that 146 human genes related to PBM and 268 genes related to PDT. The proportion of gene intersection is $39.9 \%$, and the proportion of KEGG of PBM and PDT is $78.9 \%$. Therefore, it can be concluded that the two different phototherapy methods, PBM and PDT, may regulate the same pathway to play an important biological role.

Moreover, the light sources of LED and laser were compared to verify if there are significant differences. As a result, 75 phototherapy-related genes with LED and 220 phototherapy-related genes with laser were identified. The ratio of the two intersecting genes in LED genes is $98.9 \%$, which means that most of the phototherapy related genes in LED are covered by the laser-related phototherapy genes. Therefore, it can also be proved that their effects e have no significant differences in phototherapy. For the 75 LED genes related to phototherapy, there was no KEGG result caused by few identified genes.

We speculated that the 29 genes in the PBM, PDT, LED, and laser are the key genes for the role of phototherapy. Therefore, the enrichment analysis was performed on the 29 genes to explore the mechanism of phototherapy. The result of KEGG was shown to be related to Longevity regulation, Amphetamine addiction, Melanoma, etc. GO results show that these genes were related to tyrosine, ultraviolet, light, and oxygen stress, etc. On the one hand, the results of KEGG of these 29 genes were only 7 pathways caused by few genes; on the other hand, from GO results, the function of these genes can be understood to further clarify the role of phototherapy. 
Due to the repeated occurrence of RA signaling pathways in our results, we also performed gene sequencing in MH7A cells under the conditions of whether there was light or not in TNF-a stimulation to explore the differentially expressed gene. Sequencing results showed that there were 2950 differential genes, and KEGG enriched 29 pathways. The top ten enriched pathways were Cytokine-cytokine receptor interaction, Neuroactive ligand-receptor interaction, Protein processing in the endoplasmic reticulum, Cell cycle, Viral protein interaction with cytokine and cytokine receptor, Complement and coagulation cascades, Legionellosis, DNA replication, Prion diseases, Bladder cancer. Among the 29 signaling pathways, 12 of them coincide with those obtained by PBM analysis, which are Cytokine-cytokine receptor interaction, Legionellosis, Prion diseases, Viral protein interaction with cytokine and cytokine receptor, Complement and coagulation cascades, Bladder cancer, Fluid shear stress, and atherosclerosis, Prostate cancer, MAPK signaling pathway, TNF signaling pathway, IL-17 signaling pathway, Hepatitis B. These 12 signaling pathways may be important in PBM.

A PPI network was constructed in the genes of 12 pathways to explore outstanding proteins. The network contained 129 nodes and 1235 edges. And we identified ten outstanding proteins in this network, which were IL6, TNF, VEGFA, IL 10, EGFR, EGF, CCL2, MMP9, IL 1B, FOS. And IL6 was the most important that connected with 84 nodes in the network. In fact, in our previous study, we have demonstrated the effect of 630nm LED on MH7A cells, and the IL6 decreased after 630nm LED by ELISA experiment and q-PCR. In this network, there were six clusters generated and the cluster with the highest score was chosen. In this cluster, a total of nine proteins appeared in MH7A and PBM results, and the eight proteins appeared in only LED stimulate-MH7A cells. The nine proteins (red circles) had a very high degree, which proves the feasibility of our method. The eight proteins (yellow circles), including CSF3, CXCL3, SOCS3, HMOX1, HBEGF, IL1B, IL 1A, PLAU, CCL20, represent the part of differentially expressed genes in LED stimulateMH7A. It means that they not have been studied and have research value and potential in PBM. For the accuracy of the PPI network and the effect of $640 \mathrm{~nm}$ LED red light, the RT-qPCR was used to analysis mRNA levels of inflammatory. There was a significant decrease in the gene expressions of inflammatory cytokines IL-6, IL-8, and IL-1Band MMP-3 expression; meanwhile, the expression of antiinflammatory factor IL-10 was promoted with the radiation doses increased. These results demonstrate that the gene expression and production of inflammatory cytokines by MH7A cells can be inhibited by 630 -nm LED radiation.

In this experiment, due to the limitations of the PubMed search engine, we tried to use different keywords to search for articles related to light therapy. Although a simple search will expand the number of literature, the accuracy will also decrease. However, according to the late start of LED research, the number of related literature was not much. To expand the number of literature, the simplest search formula was chosen: light-emitting diode. Accuracy was sacrificed, but in post-processing, the intersections of LED genes with those identified in PBM and PDT genes were studied. Therefore, the method can guarantee both quantity and accuracy.

In gene identification, we used the gene_atomization (a function) in the pubmed.mineR package $(\mathrm{R}$ language). But the function has one thing worth noting. In some cases, it recognizes the carbon element 
3 as C3 but enters it as the gene: complement 3. Therefore, after we used the function to identify the human gene ID, the confounding genes need to be removed artificially. During manual processing, it was found that most of the confusing genes were 2-bit, such as those of PC and TG, which could be used to focus attention on this type of gene.

\section{Conclusion}

In summary, web crawling is a promising strategy to generate study hypotheses for guiding and prioritizing future studies. Used web crawling, bioinformatics analysis, and gene sequence, we identified 12 pathways and 10 genes that play important roles in PBM and predicted nine genes with research value. Anti-inflammatory effect of $630 \mathrm{~nm}$ LED red light was demonstrated by RT-qPCR. Moreover, it was also verified that PBM and PDT, as well as LED and laser have no significant difference in effect on phototherapy.

\section{List Of Abbreviations}

PBM: photobiomodulation;

LED: ight-emitting diode;

LLLT: low-level laser therapy;

PDT: photodynamic therapy;

RT-qPCR: Real Time Quantitative PCR;

KEGG:Kyoto encyclopedia of genes and genomes;

GO: the gene ontology term

\section{Declarations}

\section{Ethics approval and consent to participate}

Not applicable.

\section{Consent for publication}

Not applicable.

\section{Availability of data and materials}

The datasets used and/or analysed during the current study are available from the corresponding authors on reasonable request. 


\section{Competing interests}

The authors declare that they have no competing interests

\section{Funding}

This work was supported by the National Major Project for the research task (no. 2017YFB040380).

\section{Authors' contributions}

Hao Wu and Caiyun Meng crawled literature contributed to data analysis and RT-qPCR. Hao Wu and Qing Xia wrote the manuscript. Wuqi Song designed the project. Wuqi Song and Fengmin Zhang reviewed the manuscript. Wuqi Song and Hailiang Liu revised the manuscript.

\section{Acknowledgements}

We thank Truwin Optoelectronic Medical (Beijing, China) for building the LED device according to our design.

\section{Authors' information (optional)}

\section{References}

1. Anders JJ, Lanzafame RJ, Arany PR. Low-level light/laser therapy versus photobiomodulation therapy. Photomed Laser Surg. 2015;33(4):183-4. doi:10.1089/pho.2015.9848.

2. Baxter GD, Liu L, Petrich S, Gisselman AS, Chapple C, Anders JJ, et al. Low level laser therapy (Photobiomodulation therapy) for breast cancer-related lymphedema: a systematic review. BMC Cancer. 2017;17(1):833. doi:10.1186/s12885-017-3852-x.

3. Santinoni CD, Oliveira HF, Batista VE, Lemos CA, Verri FR. Influence of low-level laser therapy on the healing of human bone maxillofacial defects: A systematic review. J Photochem Photobiol B. 2017;169:83-9. doi:10.1016/j.jphotobiol.2017.03.004.

4. Pinar Avci AG, Sadasivam M, Vecchio D, Pam Z, Pam N, Hamblin MR. Low-level laser (light) therapy (LLLT) in skin: stimulating, healing, restoring. Semin Cutan Med Surg. 2013;32(1):41-52.

5. Dae-Hyun Jang D-HS, Eun-Ju Chang, Jae Yong Jeon. Anti-inflammatory and lymphangiogenetic effects of low-level laser therapy on lymphedema in an experimental mouse tail model. Lasers Med Sci. 2015;31(2):289-96. doi:10.1007/s10103-015-1854-y.

6. Chen AC, Arany PR, Huang YY, Tomkinson EM, Sharma SK, Kharkwal GB, et al. Low-level laser therapy activates NF-kB via generation of reactive oxygen species in mouse embryonic fibroblasts. PLoS One. 2011;6(7):e22453. doi:10.1371/journal.pone.0022453.

7. Zhang L, Zhang Y, Xing D. LPLI inhibits apoptosis upstream of Bax translocation via a GSK-3betainactivation mechanism. J Cell Physiol. 2010;224(1):218-28. doi:10.1002/jcp.22123. 
8. Sperandio FF, Giudice FS, Correa L, Pinto DS Jr, Hamblin MR, de Sousa SC. Low-level laser therapy can produce increased aggressiveness of dysplastic and oral cancer cell lines by modulation of Akt/mTOR signaling pathway. J Biophotonics. 2013;6(10):839-47. doi:10.1002/jbio.201300015.

9. Belotto RA, Chavantes MC, Tardivo JP, Euzebio Dos Santos R, Fernandes RCM, Horliana A, et al. Therapeutic comparison between treatments for Vulvar Lichen Sclerosus: study protocol of a randomized prospective and controlled trial. BMC Womens Health. 2017;17(1):61. doi:10.1186/s12905-017-0414-y.

10. Schuitmaker JJ, van Leengoed PB,HLLM, van der Meulen FW, Star WM, van Zandwijk N. Photodynamic therapy: a promising new modality for the treatment of cancer. J Photochem Photobiol B. 1996;34(Biology):3-12.

11. Wenbo Wu DM, Hu F, Xu S, Chen C, Zhang C-J, Cheng X, Yuan Y, Ding D, Kong D, Liu B. (2017). A highly efficient and photostable photosensitizer with near-infrared aggregation-induced emission for image-guided photodynamic anticancer therapy. Advanced materials 29(33). doi:10.1002/adma.201700548.

12. Yu Cheng ACS, Joseph D Meyers,Irene Panagopoulos, and Fei B, a.C.B. (2008). Highly Efficient Drug Delivery with Gold Nanoparticle Vectors for in Vivo Photodynamic Therapy of Cancer. Journal of the American Chemical Society 130(32), 10643-10647.

13. Klebanov GI, Shuraeva N, Chichuk TV, Osipov AN, Vladimirov I. Biofizika. 2006;51(1):116-22.

14. Yu G, Wang LG, Han. Y(2012). clusterProfiler: An R package for comparing biological themes among gene clusters. OMICS,16, 284-287.

15. Szklarczyk D, Franceschini(2015).A. STRING v10: Protein-protein interaction networks, integrated over the tree of life. Nucleic Acids Research,(Database issue) 43,D447-D452.

16. Bandettini WP, Kellman P, Mancini C, Booker OJ, Vasu S, Leung SW. MultiContrast Delayed Enhancement (MCODE) improves detection of subendocardial myocardial infarction by late gadolinium enhancement cardiovascular magnetic resonance: A clinical validation study. J Cardiovasc Magn Reson. 2012;14:83.

17. Ritchie ME, Phipson B, Wu D, Hu Y, Law CW, Shi W, Smyth GK. limma powers differential expression analyses for RNA-sequencing and microarray studies. Nucleic acids research. 2015;43(7):e47. doi:10.1093/nar/gkv007.

18. Meng C, Xia Q, Wu H, Huang H, Liu H, Li Y, et al. Photobiomodulation with 630-nm LED radiation inhibits the proliferation of human synoviocyte MH7A cells possibly via TRPV4/PI3K/AKT/mTOR signaling pathway. Lasers Med Sci. 2020;35(9):1927-36. doi:10.1007/s10103-020-02977-5.

19. Gao ML, Deng WL, Huang N, Wang YY, Lei XL, Xu ZQ, et al. (2016). Upregulation of GADD45a in lightdamaged retinal pigment epithelial cells. Cell Death Discovery 2(1). doi:10.1038/cddiscovery.2016.13.

20. Li Y, Xu Q, Shi M, Gan P, Huang Q, Wang A, et al. Low-level laser therapy induces human umbilical vascular endothelial cell proliferation, migration and tube formation through activating the PI3K/Akt signaling pathway. Microvasc Res. 2020;129:103959. doi:10.1016/j.mvr.2019.103959. 
21. Souza NH, Marcondes PT, Albertini R, Mesquita-Ferrari RA, Fernandes KP, Aimbire F. Low-level laser therapy suppresses the oxidative stress-induced glucocorticoids resistance in U937 cells: relevance to cytokine secretion and histone deacetylase in alveolar macrophages. J Photochem Photobiol B. 2014;130:327-36. doi:10.1016/j.jphotobiol.2013.12.010.

22. Li H, Sun T, Liu C, Cao Y, Liu X. Photobiomodulation (450 nm) alters the infection of periodontitis bacteria via the ROS/MAPK/mTOR signaling pathway. Free Radic Biol Med. 2020;152:838-53. doi:10.1016/j.freeradbiomed.2020.01.184.

23. Neves LMS, Gonçalves ECD, Cavalli J, Vieira G, Laurindo LR, Simões RR, et al. Photobiomodulation Therapy Improves Acute Inflammatory Response in Mice: the Role of Cannabinoid Receptors/ATPSensitive K + Channel/p38-MAPK Signalling Pathway. Mol Neurobiol. 2017;55(7):5580-93. doi:10.1007/s12035-017-0792-z.

24. Son JH, Park BS, Kim IR, Sung IY, Cho YC, Kim JS, et al. A novel combination treatment to stimulate bone healing and regeneration under hypoxic conditions: photobiomodulation and melatonin. Lasers Med Sci. 2017;32(3):533-41. doi:10.1007/s10103-017-2145-6.

25. Tourassi G, Yoon H-J, Xu S, Han X. The utility of web mining for epidemiological research: studying the association between parity and cancer risk. J Am Med Inform Assoc. 2016;23(3):588-95. doi:10.1093/jamia/ocv141.

\section{Figures}




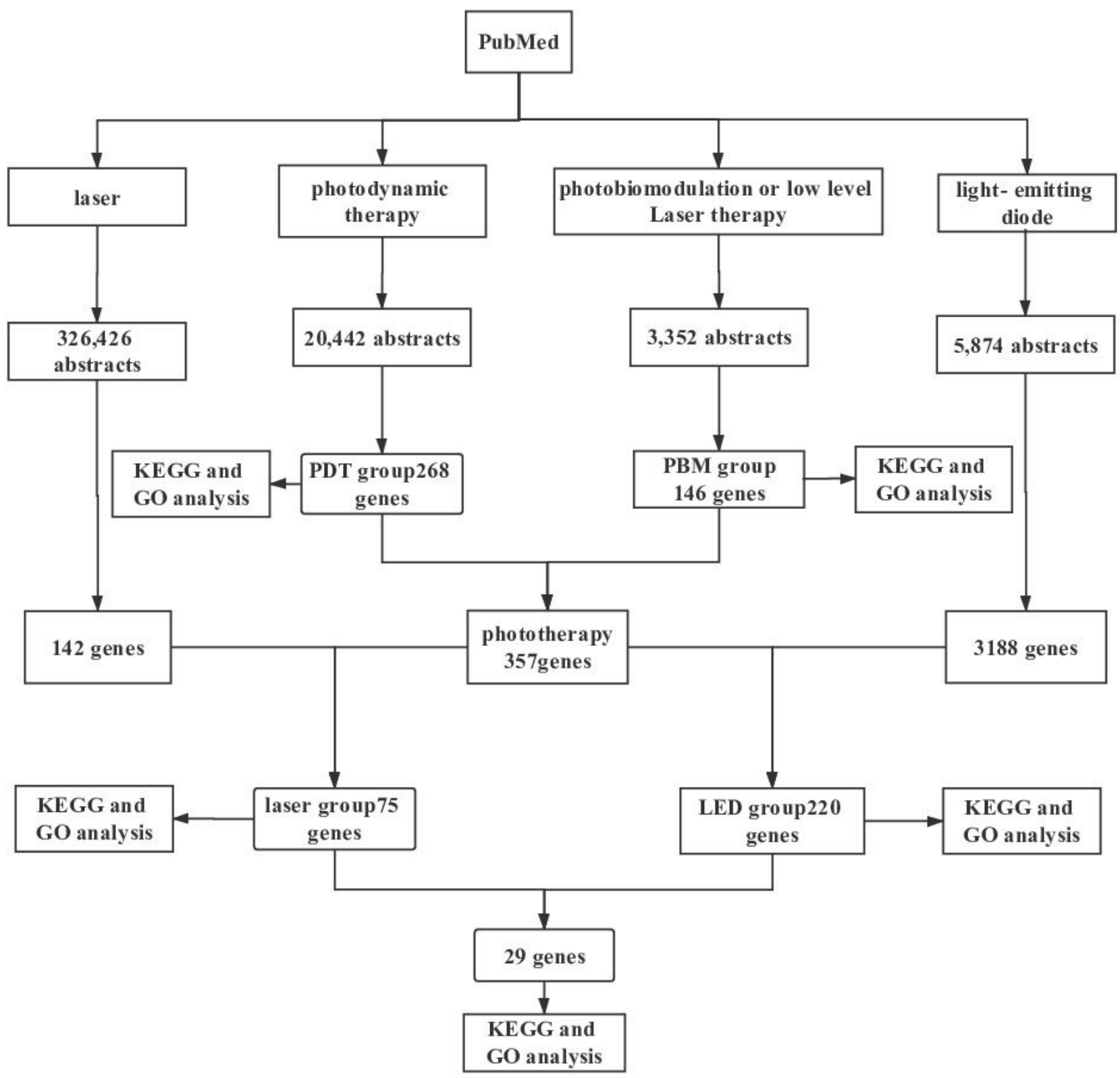

Figure 1

Data collection and analysis. 


\section{Frequency}

A

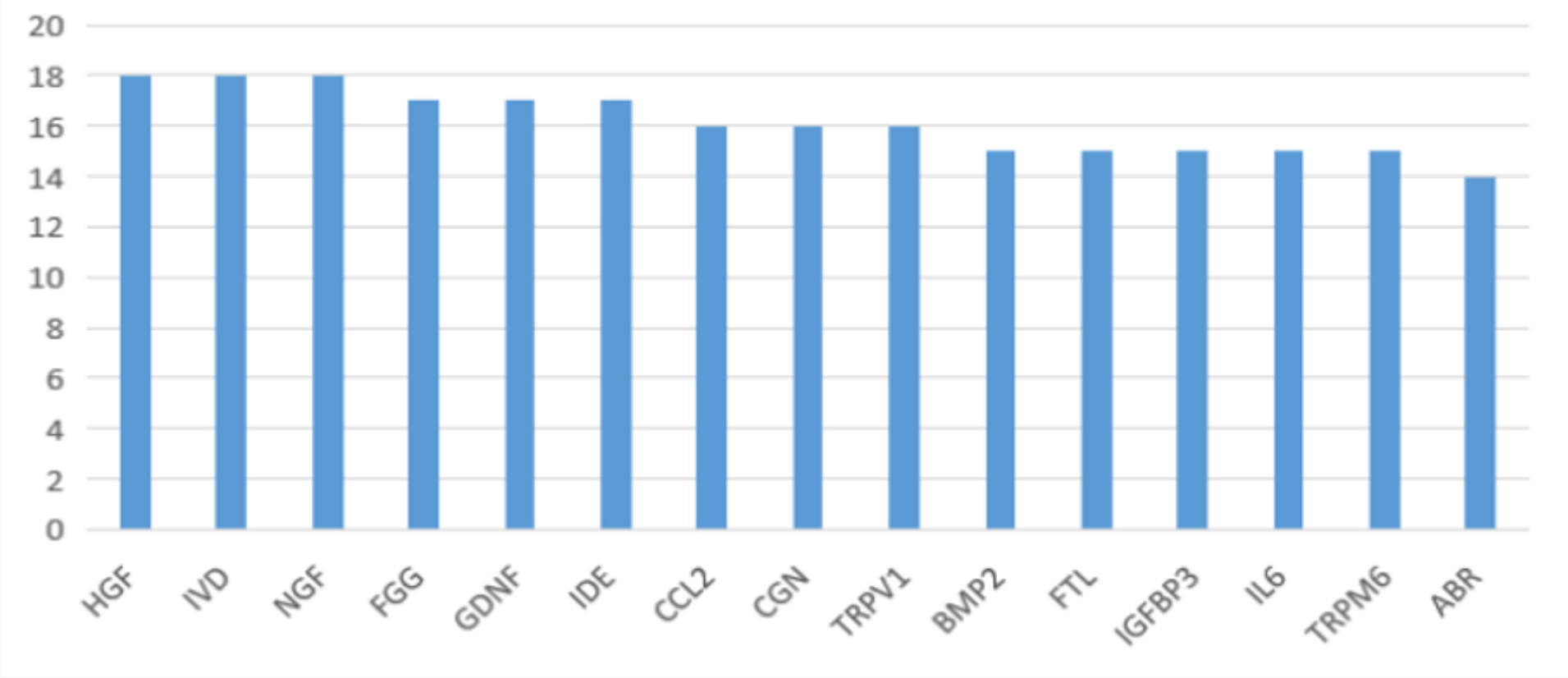

\section{Frequency}

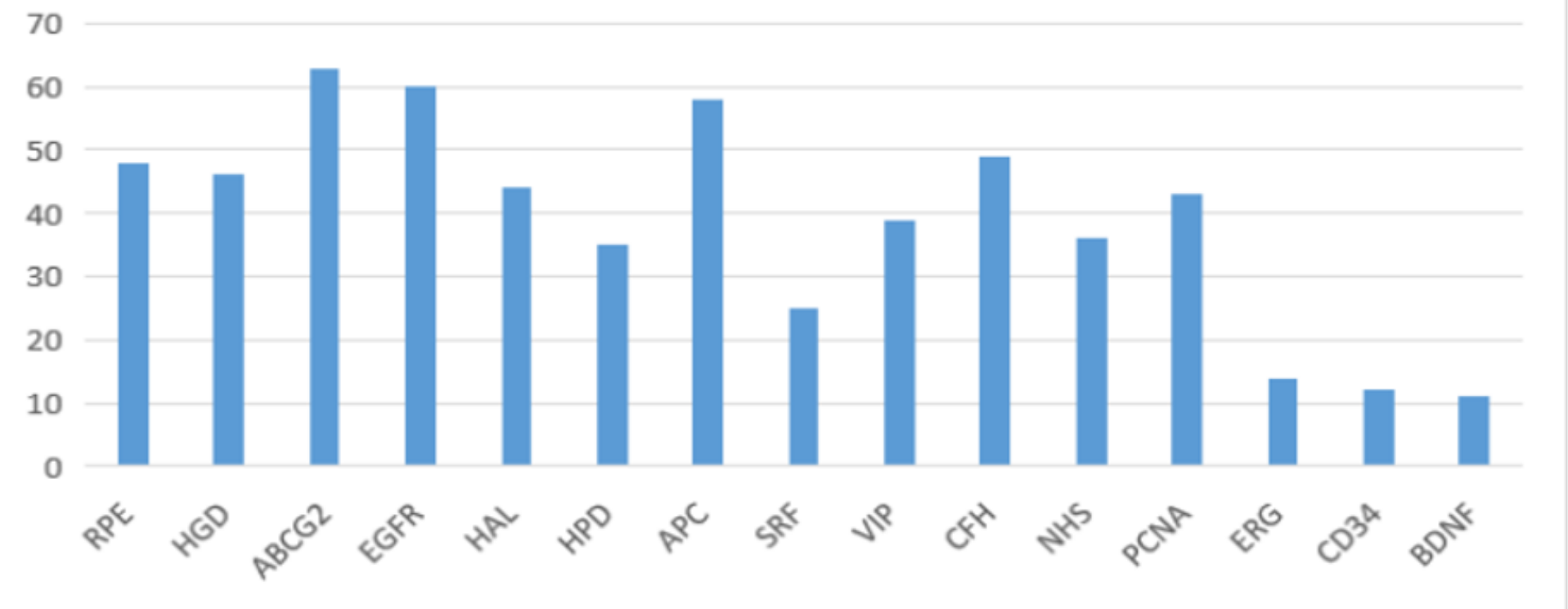

\section{Figure 2}

Frequency of the top 15 genes with the most occurrences in the PBM group(a) and the PDT group(b). 


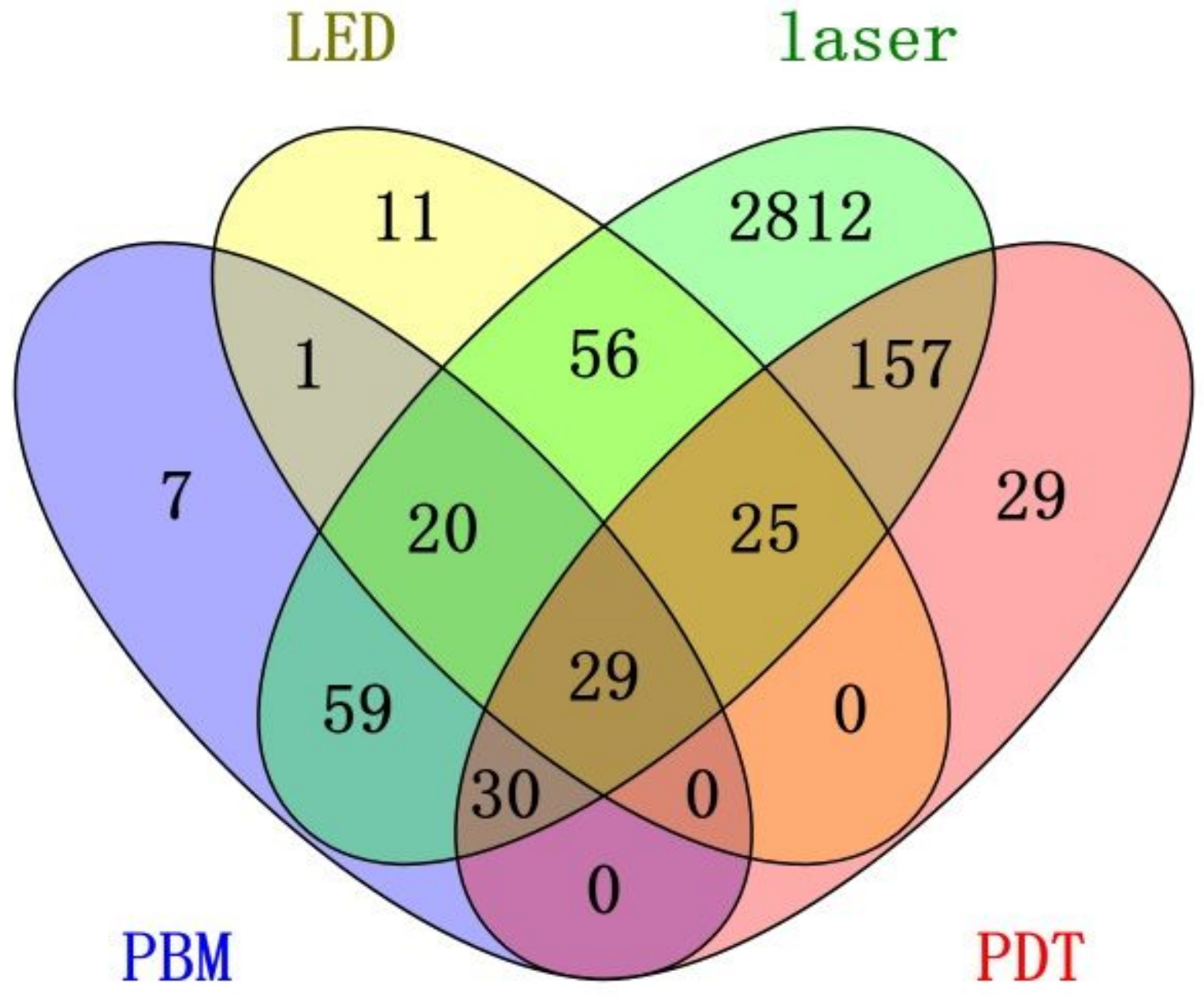

Figure 3

Venn diagram of each gene in every group. There are 161 human genes related to LED, among which 50 intersect with PBM, 54 intersect with PDT, and 29 intersect repeatedly. There are 3,188 human genes related to laser therapy, among which 138 intersect with PBM, 141 intersect with PDT, and 59 intersect repeatedly. 


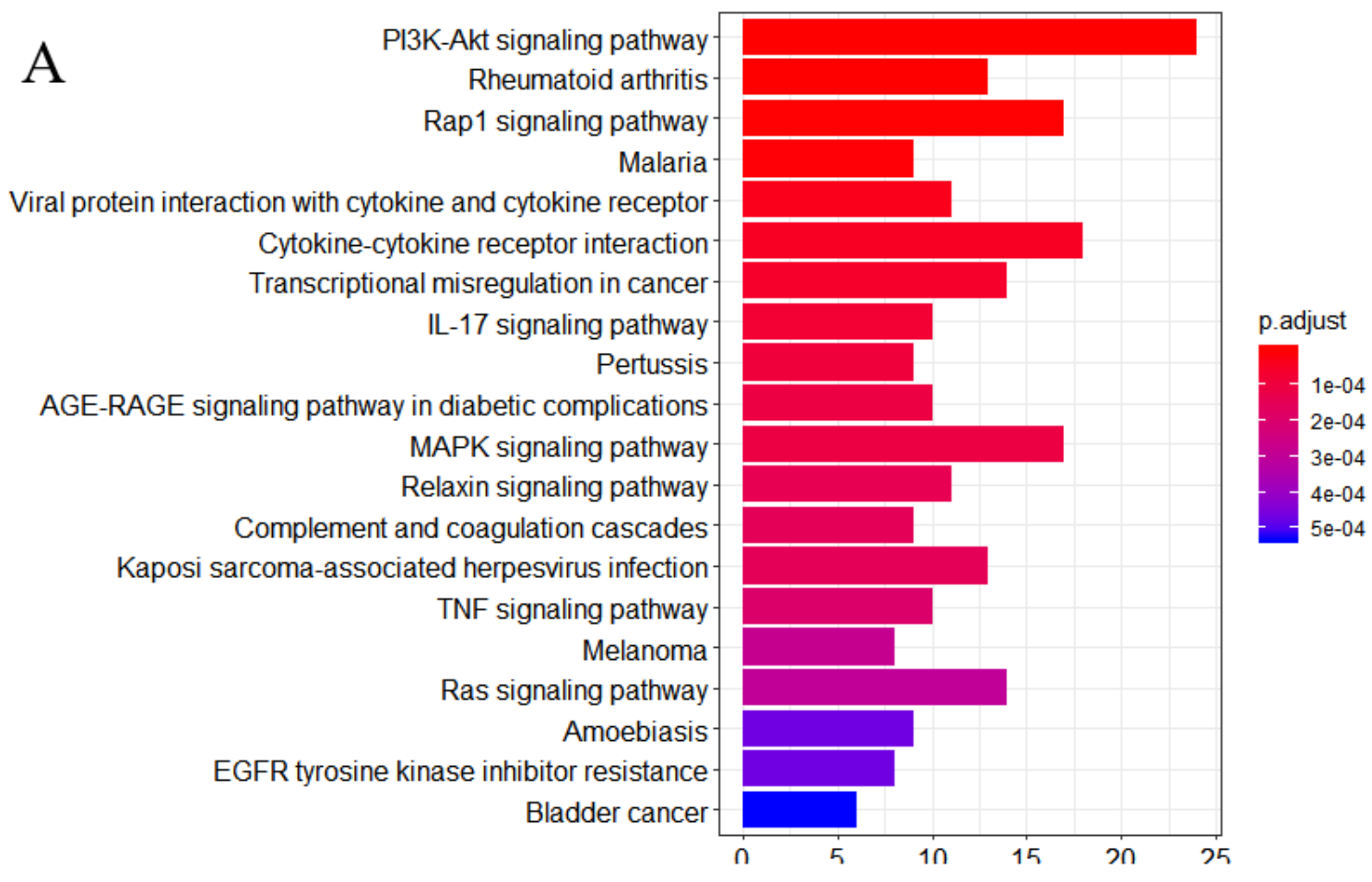

$\mathrm{B}$

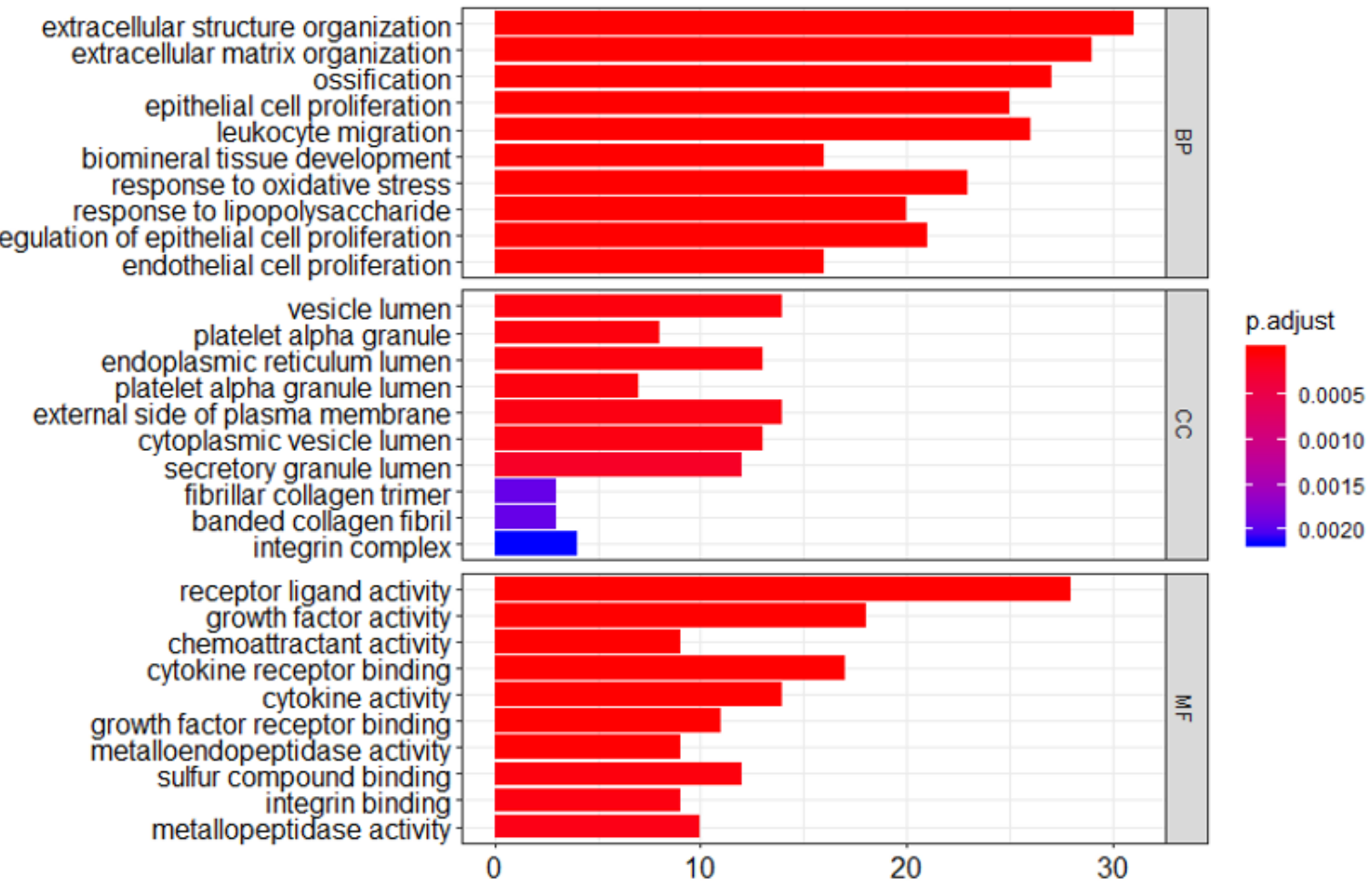

\section{Figure 4}

The top 20 KEGG results of the PBM group(a), the top 10 GO results for the PBM group(b). All KEGG and $\mathrm{GO}$ enrichment analysis was based on a threshold of $\mathrm{p}$-value $<0.05$. 
A

Proteoglycans in cancer

EGFR tyrosine kinase inhibitor resistance-

Measles

Prostate cancer

Melanoma

Bladder cancer

Epstein-Barr virus infection

Kaposi sarcoma-associated herpesvirus infection-

Endometrial cancer-

Colorectal cancer

Platinum drug resistance

FoxO signaling pathway

MAPK signaling pathway

Toll-like receptor signaling pathway

IL-17 signaling pathway

TNF signaling pathway

Human T-cell leukemia virus 1 infection

p53 signaling pathway

B

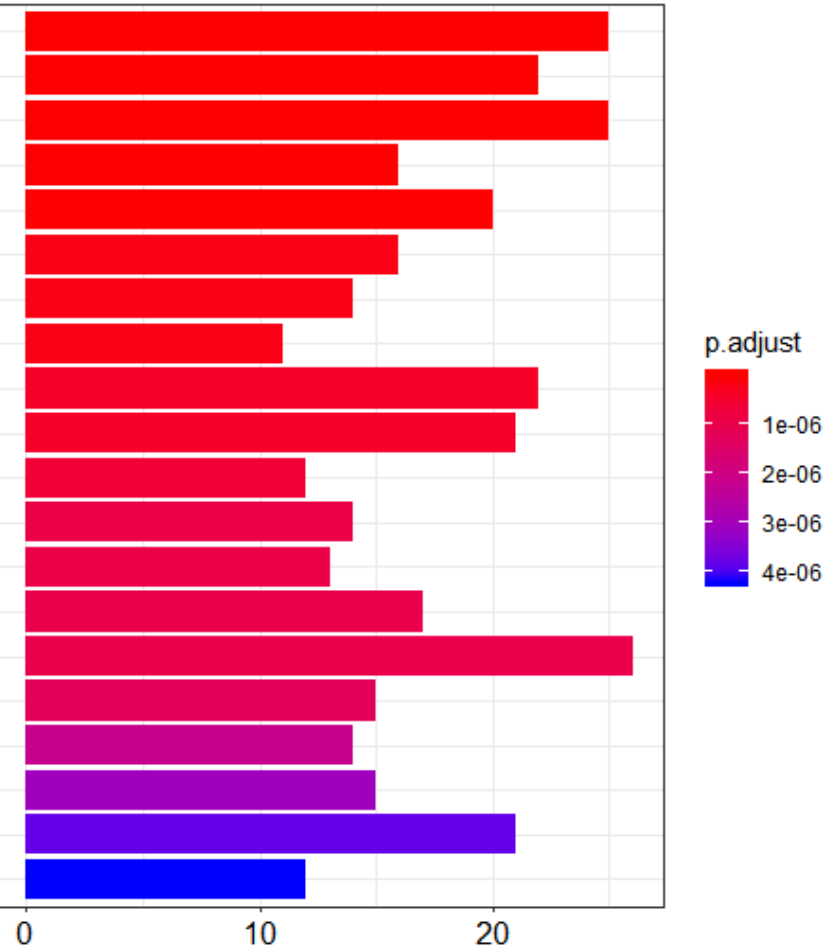

response to oxidative stress response to reactive oxygen species response to nutrient levels neuron death

cellular response to oxidative stress positive regulation of cytokine production cellular response to reactive oxygen species

cellular response to external stimulus

response to molecule of bacterial origin response to lipopolysaccharide

external side of plasma membrane vesicle lumen membrane raft membrane microdomain apical part of cell membrane region cytoplasmic vesicle lumen apical plasma membrane secretory granule lumen basolateral plasma membrane
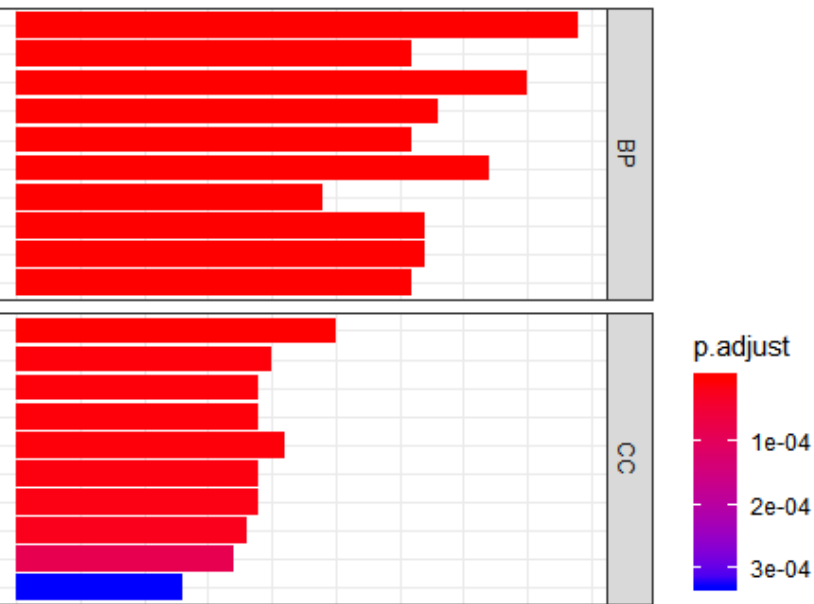

receptor ligand activity cytokine receptor binding growth factor activity

tumor necrosis factor receptor binding glycosaminoglycan binding tumor necrosis factor receptor superfamily binding growth factor receptor binding cytokine activity sulfur compound binding copper ion binding

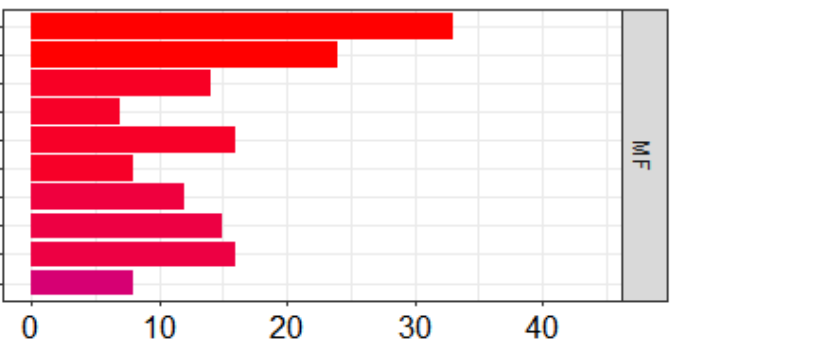

Figure 5

The top 20 KEGG results of PDT(a), top 10 GO results in PDT group(b). All KEGG pathway and GO enrichment analysis was based on a threshold of $p$-value $<0.05$. 


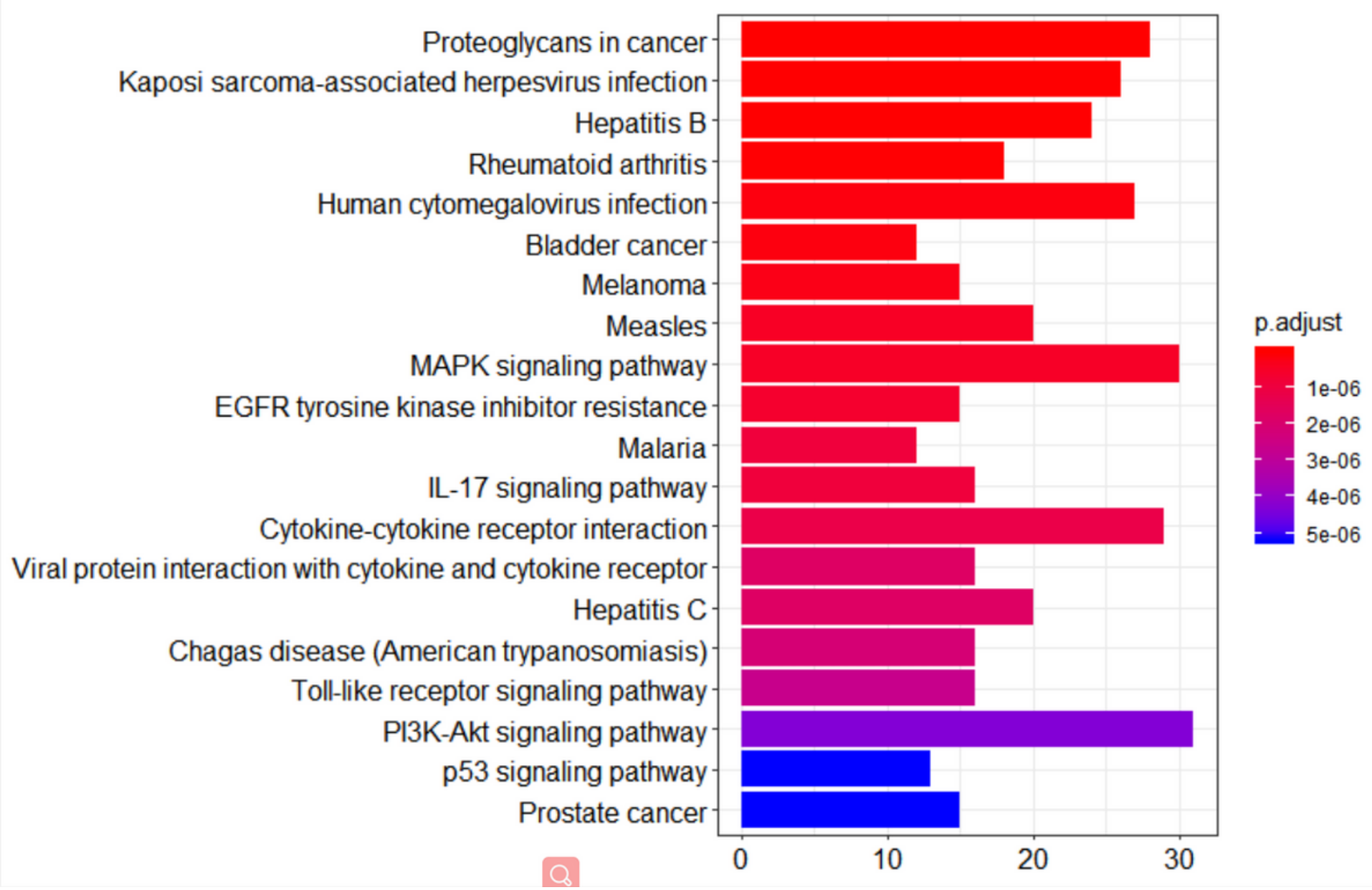

\section{Figure 6}

The top 20 KEGG results of laser group. All KEGG pathway and GO enrichment analysis was based on a threshold of $p$-value $<0.05$. 
A

Longevity regulating pathway

Amphetamine addiction

Melanoma

EGFR tyrosine kinase inhibitor resistance

Transcriptional misregulation in cancer
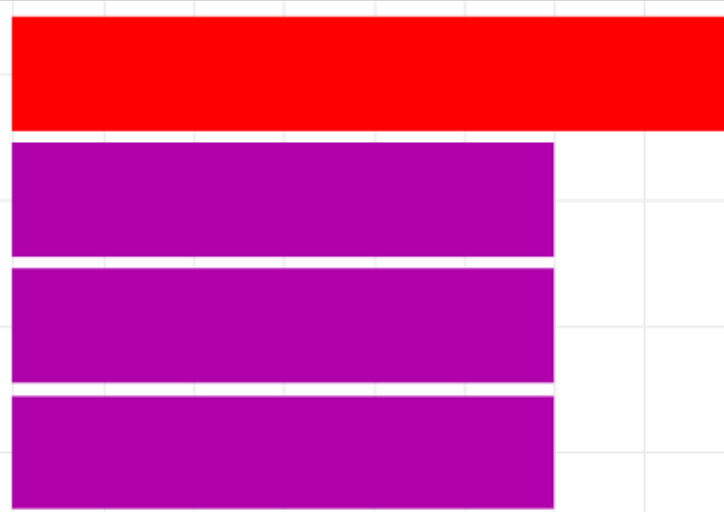

p.adjust

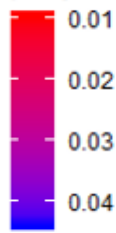

Toll-like receptor signaling pathway

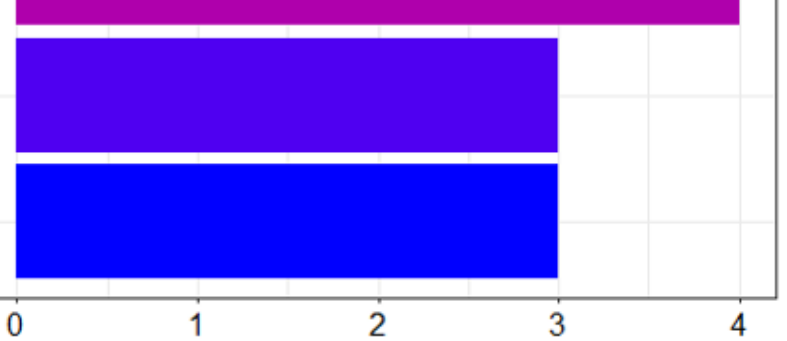

Prostate cancer -

B

positive regulation of tumor necrosis factor superfamily cytokine production
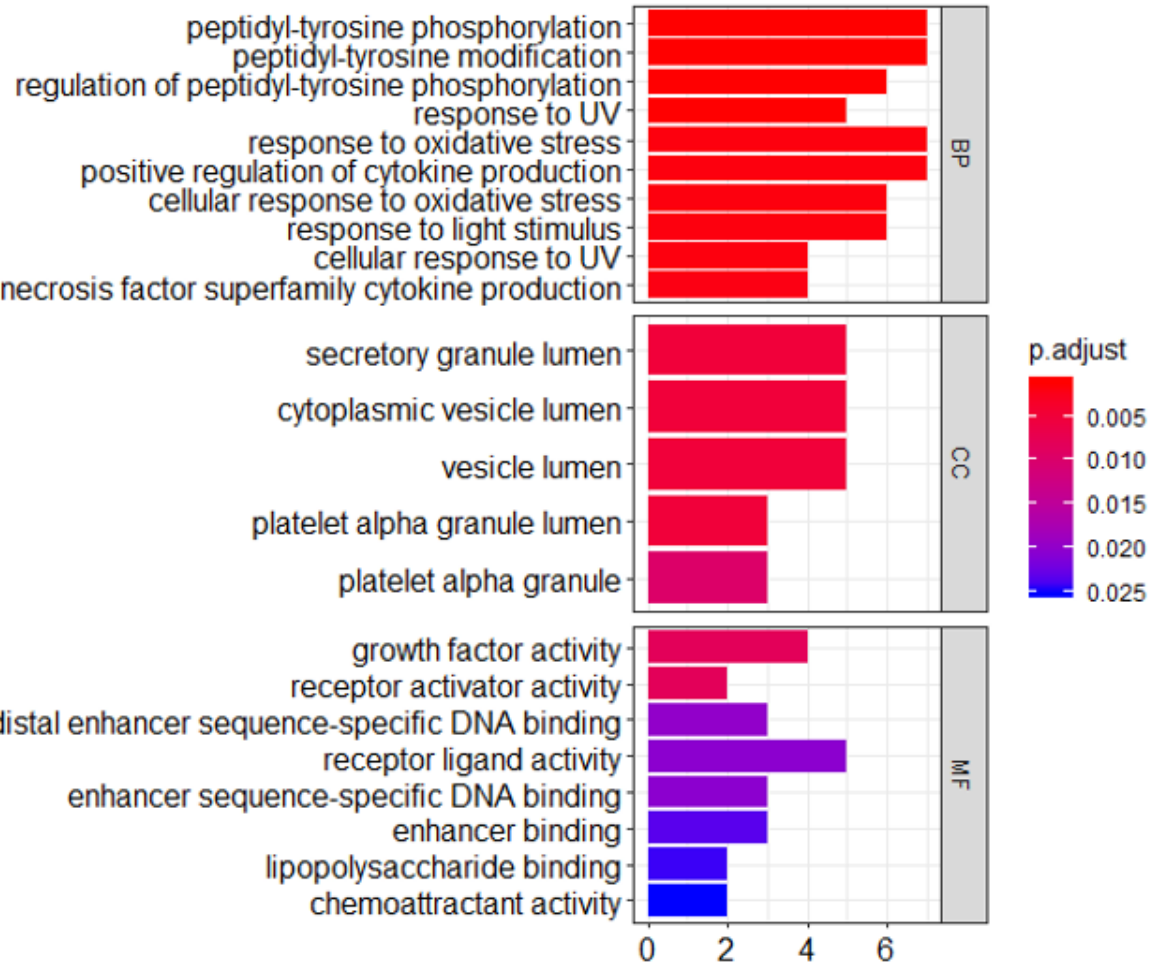

\section{Figure 7}

KEGG results of 29 common intersection genes(a), the top ten $\mathrm{GO}$ results of 29 common intersection genes(b). All KEGG pathway and GO enrichment analysis was based on a threshold of $p$-value $<0.05$. 


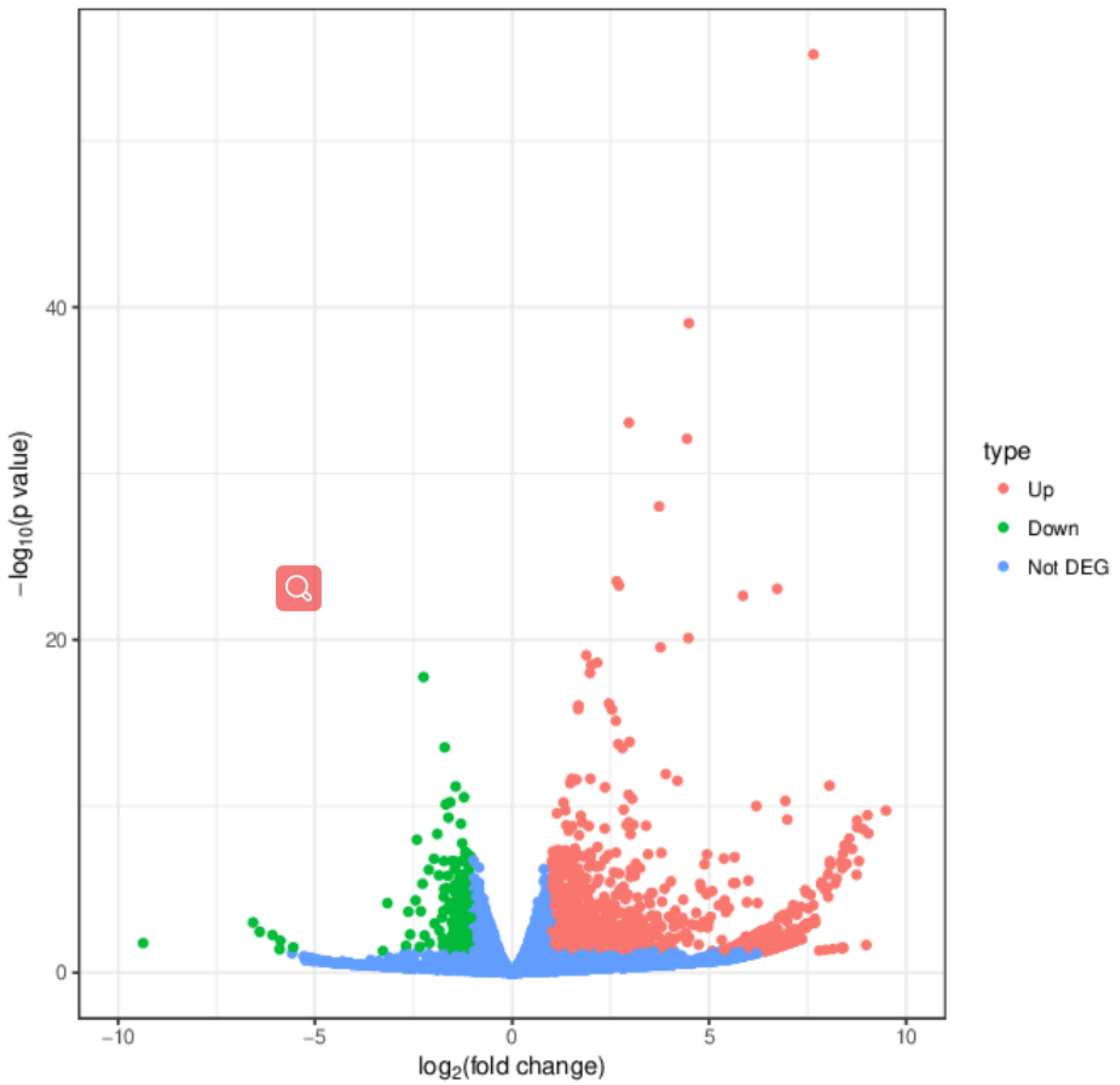

Figure 8

the volcano of differentially expressed genes in MH7A cells. A total of 2950 differentially expressed genes were identified.1885 and 1065 differentially expressed genes are up-regulated, down-regulated, respectively. P-value $<0.05$ and $\mid$ log2fold change $(F C) \mid>1$, were chosen as the cut-off threshold. 


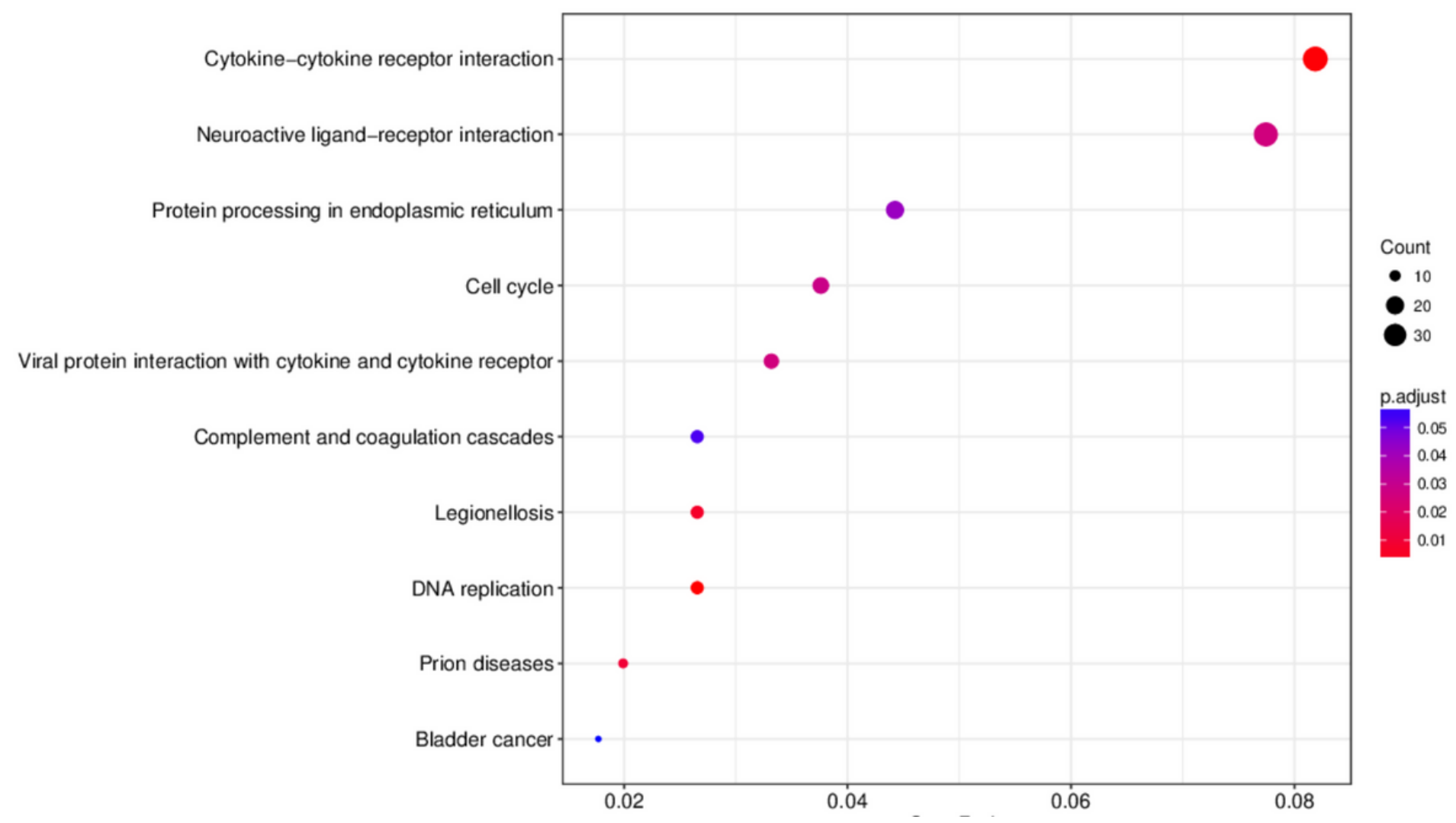

Figure 9

The top 10 KEGG results of differentially expressed genes in MH7A cells. All KEGG analysis was based on a threshold of $p$-value $<0.05$. 


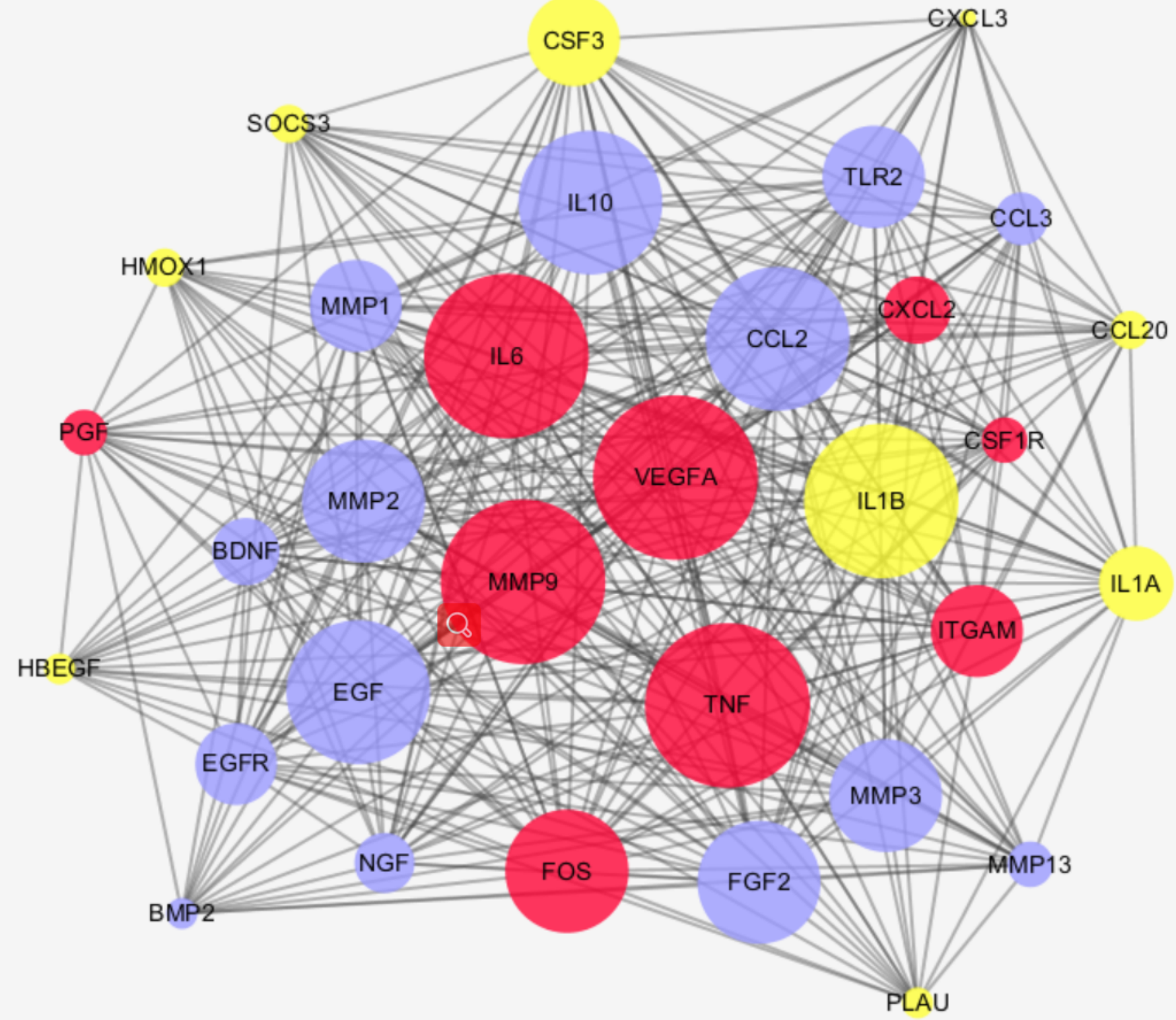

Figure 10

The top one hub modules of the PPI network evaluated in MOCDE. MCODE1 contained 32 nodes and 359 edges. The blue circles represent PBM genes, the blue circles represent MH7A genes, and red circles represent the genes repeated PBM and MH7A. The circle size represents the degree. 


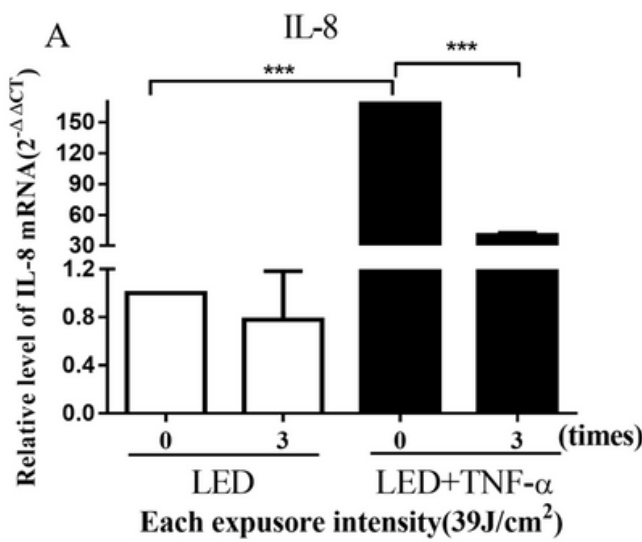

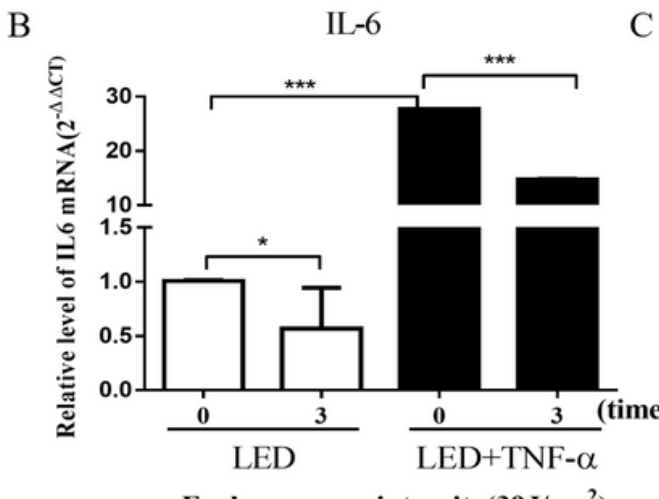

Each expusore intensity $\left(39 \mathrm{~J} / \mathrm{cm}^{2}\right)$

E

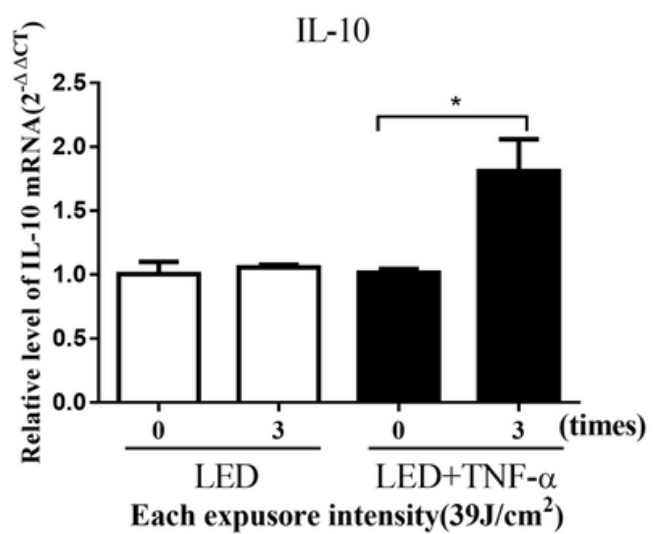

Each expusore intensity $\left(39 \mathrm{~J} / \mathrm{cm}^{2}\right)$
MMP-3

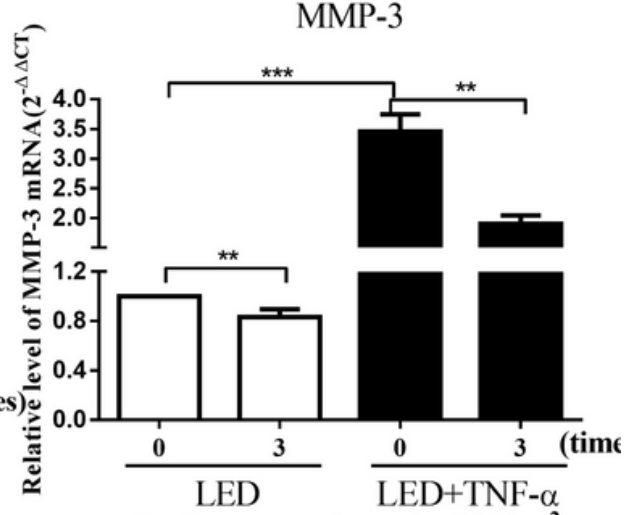

Each expusore intensity $\left(39 \mathrm{~J} / \mathrm{cm}^{2}\right)$

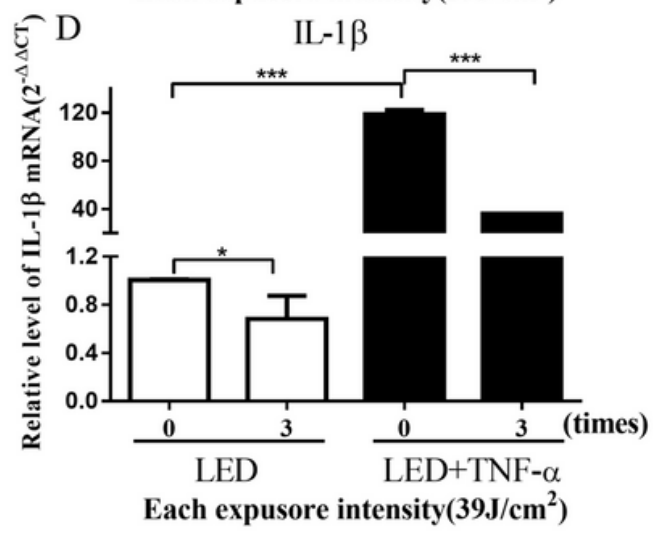

\section{Figure 11}

The effects of 630-nm LED radiation on inflammatory cytokines in synoviocyte MH7A cells at varying light doses. RT-qPCR showed changes in the relative expression levels of IL-8(a), IL-6(b), MMP3(c), IL1B(d), and IL10 (e) in MH7A cells. 